\title{
Forças no sistema de referência acelerado de um pêndulo: estudo teórico e resultados experimentais
}

Forces in the accelerated reference system of a pendulum: theoretical study and experimental results

\author{
Camila Brito Collares da Silva ${ }^{*}$, Bruno Borges Fagundes ${ }^{1}$, Januário Dias Ribeiro ${ }^{1}$, Arlei Prestes \\ Tonel $^{1}$, Fernando Lang da Silveira ${ }^{2}$, Pedro Fernando Dorneles \\ ${ }^{1}$ Universidade Federal do Pampa, Bagé, RS, Brasil \\ ${ }^{2}$ Universidade Federal do Rio Grande do Sul, Porto Alegre, RS, Brasil
}

\begin{abstract}
Recebido em 13 de Abril de 2019. Revisado em 30 de Agosto de 2019. Aceito em 19 de Outubro de 2019.
Neste trabalho realizamos, inicialmente, uma discussão teórica sobre as forças no sistema de referência acelerado de um pêndulo que oscila livre de resistência. Tal discussão propicia a afirmação de que, se um acelerômetro for fixado no centro de oscilação de um pêndulo, ele deverá registrar um valor variável sobre o eixo orientado radialmente e um valor nulo no eixo alinhado tangencialmente ao movimento do pêndulo. Para ilustrarmos aplicações dessa afirmação em duas situações de simples verificação, apresentamos duas questões instigantes: a) o que acontecerá com a bolha de ar de um nível de pedreiro, se posicionarmos o nível na direção tangencial enquanto nos embalamos em um balanço de uma praça?; b) se colocarmos para oscilar em um balanço uma pequena esfera, ela irá ficar em repouso em relação balanço? As respostas contraintuitivas às duas questões são de que tanto a bolha no nível de pedreiro quanto a esfera permanecem em repouso em relação ao balanço se posicionadas em seu centro de oscilação. Com o intuito de elucidarmos essas duas questões, concebemos um aparato experimental composto por um pêndulo físico, no qual anexamos uma placa microcontrolada Arduino, um sensor acelerômetro e um módulo Bluetooth.
\end{abstract}

Palavras-chave: Pêndulo, Forças não inerciais, Acelerômetro, Arduino.

In this work we initially lead a theoretical discussion about the forces acting on a physical pendulum oscillating without friction in an accelerated reference system. Such discussion allows us the statement that if an accelerometer is fixed on the center of oscillation of the pendulum, it will register an oscillating value in the radial axis and a null value in the tangential axis. To illustrate this proposition, we will present two simple situation for verification and then we inquire two instigating questions: a)What will happen to the air bubble of a mason level, if we position the same in the tangential direction while we cradle in a swing of a square? b) If we put a small sphere on oscillating swing, will the sphere be in rest during the oscillation? The answers for this counter intuitive questions are that both, the air bubble and small sphere, will stay in rest in relation to the swing if they are positioned on the center of oscillation of the pendulum. In order to elucidate the above questions, we designed an experimental apparatus composed of a physical pendulum, in which we attached an Arduino microcontroller board, an accelerometer sensor and a Bluetooth module.

Keywords: Pendulum, Non-inertial forces, Accelerometer, Arduino.

\section{Introdução}

Em uma recente revisão da literatura foi identificado um número significativo de publicações (20 artigos) sobre a plataforma microcontrolada Arduino, no período de 2013 a 2017 [1]. Tais artigos foram categorizados em propostas aplicadas em sala de aula e propostas para aplicação em sala de aula. A categoria predominante foi a de propostas para aplicações, com 17 trabalhos, e somente três trabalhos envolveram aplicações reais em sala de aula.

Dentre as propostas para aplicações, destaca-se como justificativa a quase inexistente abordagem no ensino mé-

*Endereço de correspondência: camilabcollares@gmail.com. dio dos temas envolvidos, tais como espectroscopia, efeito fotoelétrico e, principalmente, a instrumentação para a transmissão e recepção de sinais por meio de radiação infravermelha, entre outros. Sobre as aplicações em sala de aula foram obtidos resultados de pesquisa satisfatórios, porém, pontuais. Os autores concluem que, embora haja muitas propostas didáticas inovadoras, ainda há uma lacuna de trabalhos que mostram dados quanto à aprendizagem de conceitos físicos [1].

No presente trabalho, apresentamos uma abordagem teórica sobre as forças no sistema de referência acelerado de um pêndulo que oscila livre de resistência. A motivação emergiu a partir de análises teóricas e experimentais sobre dados coletados com um acelerômetro em ambiente 
de sala de aula. Durante uma aplicação didática de uma dissertação de mestrado profissional [2], identificamos que para uma interpretação física dos dados fornecidos por um acelerômetro acoplado a um pêndulo físico tornase necessária uma abordagem sobre referencial acelerado e um enfoque detalhado sobre o funcionamento de um acelerômetro. Dessa forma, apresentamos aqui uma proposta didática com aplicações reais em sala de aula, com o intuito de complementar a constatação feita por Moreira, Romeu, Alves e Silva [1] de que o baixo número de trabalhos com aplicações, além de apresentar uma lacuna sobre dados de aprendizagem de conceitos físicos, não possibilita que novas propostas de aplicações sejam geradas a partir de situações reais de sala de aula.

No trabalho de Collares da Silva [2], identificamos a necessidade de um aprofundamento teórico e experimental justificando a afirmação de que se um acelerômetro for fixado no centro de oscilação de um pêndulo, ele deverá registrar um valor variável sobre o eixo orientado radialmente e um valor nulo no eixo alinhado tangencialmente ao movimento do pêndulo. Para ilustrarmos aplicações dessa afirmação em duas situações de simples verificação, apresentamos duas questões desafiadoras, quais sejam: a) o que acontecerá com a bolha de ar de um nível de pedreiro, se posicionarmos o nível na direção tangencial enquanto nos embalamos em um balanço de uma praça (Figura 1)?; b) se colocarmos para oscilar em um balanço uma pequena esfera, ela irá ficar em repouso em relação balanço?

As respostas a essas questões intrigantes serão apresentadas após uma discussão teórica sobre as forças no sistema de referência acelerado (não inercial) de um pêndulo que oscila livre de resistência, seguidas de uma abordagem sobre o princípio de funcionamento de um

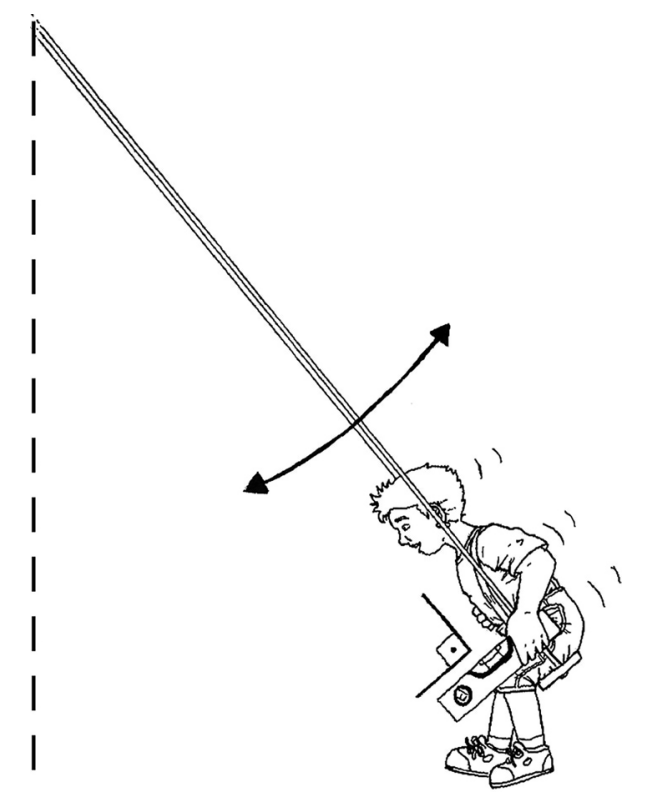

Figura 1: Ilustração de uma criança se embalando com um nível de pedreiro alinhado tangencialmente à trajetória do balanço. acelerômetro e a realização de uma análise comparativa entre as previsões teóricas e os dados experimentais coletados em sala de aula.

\section{Forças no sistema de referência acelerado de um pêndulo que oscila livre de resistência}

\subsection{Acelerações em um ponto de um pêndulo no sistema de referência da Terra}

Nesta seção, determinam-se as componentes da aceleração que um particular ponto do pêndulo físico possui quando oscila. Consideremos um pêndulo físico que pode oscilar livre de forças de resistência em torno de um eixo de suspensão O. Na Figura 2 estão indicados, além do eixo $\mathrm{O}$, o centro de massa do pêndulo $(\mathrm{CM})$ e um ponto (Q) situado a uma distância $\mathrm{r}$ do eixo de rotação. $\mathrm{O} \theta$ mede o ângulo entre o segmento de reta $\overline{O Q}$ em relação à vertical.

A resultante dos torques $\left(T_{R}\right)$, em relação ao ponto $\mathrm{O}$, é o torque devido à componente da força gravitacional $(M g \operatorname{sen} \theta)$ sendo dado por:

$$
\mathrm{T}_{R}=M g D \operatorname{sen} \theta
$$

sendo $M$ a massa do pêndulo, $g$ a aceleração gravitacional e $D$ a posição do centro de massa medido a partir do eixo de rotação.

A resultante dos torques é expressa também como:

$$
\mathrm{T}_{R}=I_{o} \frac{a_{t}}{r}
$$

sendo $I_{o} \mathrm{O}$ momento de inércia do pêndulo em relação ao eixo $\mathrm{O}$ e $a_{t}$ o valor da aceleração do ponto $\mathrm{Q}$ na direção perpendicular à direção OQ (aceleração tangente à trajetória circular do ponto $\mathrm{Q}$ em torno do ponto $\mathrm{O}$ ) e $r$, em acordo com a Figura 2, é a distância entre os pontos $\mathrm{O}$ e $\mathrm{Q}$.

Das equações (1) e (2) decorre que

$$
a_{t}=\frac{M D r}{I_{o}} g \operatorname{sen} \theta \text {. }
$$

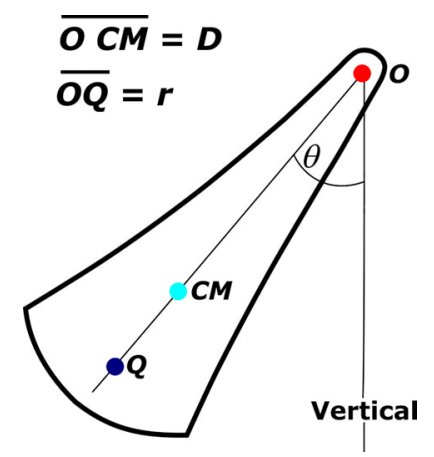

Figura 2: Pêndulo físico que oscila sem resistências em torno do eixo $\mathrm{O}$. 
Note que, no centro de massa $(r=D)$, a aceleração tangencial, usando-se o teorema de Steiner (teorema dos eixos paralelos) [3], é dada por

$$
a_{t}^{C M}=\frac{1}{1+\frac{I_{C M}}{M D^{2}}} g \operatorname{sen} \theta .
$$

Portanto, a aceleração tangencial do centro de massa do pêndulo físico é menor que gsen $\theta$, que é a aceleração tangencial do pêndulo simples.

No que segue, estaremos interessados em localizar a posição do ponto $\mathrm{Q}$ para o qual a aceleração tangencial $\left(a_{t}\right)$ seja igual ao componente da aceleração da gravidade $g$ nessa mesma direção tangente à trajetória. Esse ponto é o centro de oscilação do pêndulo físico [4] e a distância que o separa de $\mathrm{O}$ é o comprimento $L$ do pêndulo simples equivalente ao pêndulo físico. Ou seja, consideremos na equação (3) que $a_{t}=g \operatorname{sen} \theta, \operatorname{logo} r$ assume o valor $L$ dado por

$$
r\left(a_{t}=g \operatorname{sen} \theta\right)=L=\frac{I_{o}}{M D} .
$$

Expressando-se, em acordo com o teorema de Steiner, o momento de inércia em torno do ponto $\mathrm{O}$ em função do momento de inércia em torno do $\mathrm{CM}\left(I_{C M}\right)$, encontra-se que

$$
L=\frac{I_{C M}+M D^{2}}{M D}=\frac{I_{C M}}{M D}+D .
$$

Decorre da equação (6) que o centro de oscilação (ponto onde ocorre uma aceleração tangente à trajetória que é exatamente igual em valor à componente da aceleração da gravidade), está mais distante do eixo de oscilação que o centro de massa do pêndulo $(L>D)$. É fácil provar, por exemplo, que se o pêndulo é uma haste homogênea com comprimento $C$, que oscila em torno de uma das suas extremidades, o seu centro de oscilação se situa a 2C/3 do ponto O. Já para um pêndulo simples, onde $I_{C M}=0, L=D$.

Busca-se a seguir a determinação da aceleração centrípeta do ponto Q. Se o pêndulo inicia seu movimento a partir do repouso em uma posição angular afastada da vertical por um ângulo $\theta_{0}$ (Figura 3 ), ao atingir a posição angular $\theta$, tem sua energia cinética $\mathrm{K}$ dada por

$$
K=\frac{I_{0}}{2}\left(\frac{v}{r}\right)^{2}
$$

em que $v$ é a velocidade tangencial, no sistema de referência da Terra, do ponto situado na posição Q, enquanto a variação da energia potencial gravitacional do pêndulo $\left(\Delta E_{g}\right)$ ao se deslocar entre as duas posições angulares é dada por

$$
\Delta E_{g}=M g D\left(\cos \theta_{o}-\cos \theta\right)
$$

pela conservação da energia mecânica do pêndulo, ao atingir a posição angular $\theta$, a energia cinética é dada por

$$
K=-\Delta E_{g}
$$

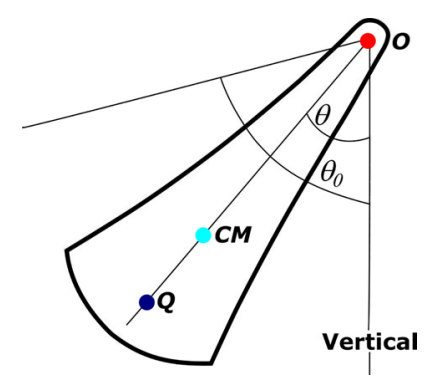

Figura 3: Pêndulo inicialmente em repouso adquire energia cinética ao passar da posição angular inicial $\theta_{0}$ para a posição angular $\theta$.

Substituindo-se (7) e (8) em (9) encontra-se

$$
\frac{v^{2}}{r}=2 \frac{M D r}{I_{0}} g\left(\cos \theta-\cos \theta_{o}\right) .
$$

No lado esquerdo da equação 10 , identifica-se a aceleração centrípeta do ponto Q.

Dessa forma, fica demonstrado que em um ponto distante $r$ do eixo de rotação as acelerações, respectivamente nos eixos orientados na direção OQ (direção radial ou centrípeta) e na direção perpendicular a $\mathrm{OQ}$, paralela à trajetória do pêndulo (direção tangencial), são:

$$
a_{c}=2 k g\left(\cos \theta-\cos \theta_{o}\right)
$$

$\mathrm{e}$

$$
a_{t}=k g \operatorname{sen} \theta
$$

em que definimos a quantidade adimensional $k=\frac{M D r}{I_{o}}=$ $\frac{r}{L}$ que expressa a razão entre a distância do ponto $Q$ em relação ao eixo de rotação (eixo de passa pelo ponto $\mathrm{O}$ ) e o comprimento $L$ do pêndulo simples equivalente (ver equação (5)).

Observe que a aceleração tangencial não depende da posição angular inicial (ver equação $(12)$ ), enquanto a aceleração centrípeta é dependente dessa posição angular inicial e também da posição do ponto Q (ver equação (11).

Como caso particular das equações (11) e (12), no centro de oscilação $(r=L$ ou $k=1)$, as acelerações, respectivamente nas direções centrípeta e tangencial, valem

$$
a_{c}=2 g\left(\cos \theta-\cos \theta_{o}\right)
$$

$\mathrm{e}$

$$
a_{t}=g \operatorname{sen} \theta
$$

\subsection{Forças sobre um pequeno objeto solidário ao pêndulo}

Aplicando a segunda lei de Newton a uma partícula em um sistema de referência $\mathrm{S}$ inercial, para um movimento de translação, a resultante das forças é dada por $\overrightarrow{F_{\text {res }}}=m \vec{a}$, sendo $\mathbf{S}^{\prime}$ um sistema de referência que possui em relação a $\mathbf{S}$ aceleração $\vec{\alpha}$, sendo $\overrightarrow{a^{\prime}}$ a aceleração 
da partícula no sistema $\mathbf{S}^{\prime}$, temos $\vec{a}=\vec{\alpha}+\overrightarrow{a^{\prime}}$, e, portanto, podemos reescrever a igualdade $\overrightarrow{F_{\text {res }}}=m \vec{a}$ como $\overrightarrow{F_{r e s}}-m \vec{\alpha}=m \overrightarrow{a^{\prime}}$. Daí, definindo $\overrightarrow{F_{r e s}^{\prime}}$ como $\overrightarrow{F_{r e s}}-m \vec{\alpha}$, obtemos $\overrightarrow{F_{r e s}^{\prime}}=m \overrightarrow{a^{\prime}}$. Ou seja, temos uma igualdade com a forma da segunda lei de Newton, mas aplicada a um sistema de referência acelerado e, portanto, não inercial. Podemos fazer uso dessa igualdade, mas observando que há em seu membro esquerdo a "força inercial ou fictícia- $m \vec{\alpha}$. A propósito, pelo "princípio da equivalência"de Einstein é como se o campo gravitacional tivesse sido alterado de $\vec{g}$ para $\vec{g}-\vec{\alpha}$.

Considerando-se um pequeno objeto de massa $m$ localizado na posição Q (Figura 3), o diagrama de forças sobre o pequeno objeto visto no sistema de referência da Terra (considerado como referencial inercial) está dado na Figura 4 . A força gravitacional $\left(\overrightarrow{F_{g}}\right)$ já está representada por suas componentes nas direções OQ (componente radial) e perpendicular a OQ (componente tangencial). A força $\vec{F}$ é a força de vínculo do objeto com o pêndulo, apresentando componentes na direção centrípeta $\left(F_{c}\right)$ e tangencial $\left(F_{t}\right)$.

Adotando-se um sistema de referência solidário ao pêndulo (referencial não inercial), ocorrerão, além das forças reais já indicadas na Figura 4, duas forças inerciais (ou fictícias) decorrentes de que o sistema de referência é não inercial. O diagrama das forças no sistema de referência do pêndulo está posto na Figura 5.

As forças inerciais, conforme representadas na Figura 5, ocorrem na mesma direção das acelerações do sistema de referência solidário ao pêndulo, mas em sentidos contrários a tais acelerações [4]. Uma dessas duas forças é a força inercial centrífuga, cujo valor é dado por

$$
F_{i, c}=m a_{c}
$$

O módulo da aceleração centrífuga é o mesmo que o dado na equação (11). Portanto, a força inercial centrífuga, expressa em função da quantidade adimensional $k$, vale

$$
F_{i, c}=2 m k g\left(\cos \theta-\cos \theta_{o}\right) .
$$

O módulo da aceleração tangencial é o mesmo que o dado pela equação 12 . Desse modo, a força inercial na

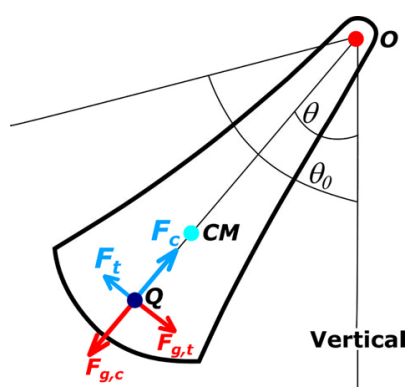

Figura 4: Forças sobre um pequeno objeto vinculado ao pêndulo no ponto Q. $F_{g, t}$ e $F_{g, c}-$ componentes na direção tangencial e centrípeta da força gravitacional. $F_{t}$ e $F_{c}$-componentes na direção tangencial e centrípeta da força de vínculo.

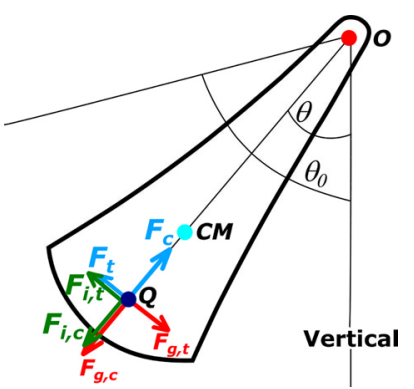

Figura 5: Diagrama das forças exercidas em um pequeno objeto no ponto $Q$ no sistema de referência não inercial solidário ao pêndulo. $F_{g, t}$ e $F_{g, c}$ - componentes na direção tangencial e centrípeta da força gravitacional. $F_{i, t}$ e $F_{i, c}$-componentes na direção tangencial e centrípeta da força inercial.

direção tangencial é

$$
F_{i, t}=m k g \operatorname{sen} \theta
$$

O pequeno objeto está em repouso em relação ao pêndulo e, portanto, nesse sistema de referência não inercial a resultante das forças sobre ele se anula na direção tangencial e na direção radial. Assim sendo, na direção tangencial a condição de repouso para o pequeno objeto, em relação ao referencial não inercial, é

$$
F_{g, t}-F_{t}-F_{i, t}=0 .
$$

A partir da projeção da força gravitacional na direção tangencial $\left(F_{g, t}=m g \operatorname{sen} \theta\right)$ e da força fictícia dada pela equação (17), encontramos a componente tangencial da força de vínculo, que é dada por

$$
F_{t}=(1-k) m g \operatorname{sen} \theta
$$

em que $m$ é a massa do pequeno objeto solidário ao pêndulo.

Na direção centrípeta ou radial, a condição de repouso do pequeno objeto em relação ao referencial acelerado é

$$
F_{c}-F_{g, c}-F_{i, c}=0 \text {. }
$$

Substituindo-se o valor da componente radial da força gravitacional $\left(F_{g, c}=m g \cos \theta\right)$ e da força centrífuga $F_{i, c}$ dada pela equação (16), encontra-se para a componente na direção radial da força de vínculo

$$
F_{c}=m g\left((2 k+1) \cos \theta-2 k \cos \theta_{o}\right) .
$$

Particularizando-se as equações (19) e 21 para o centro de oscilação $(r=L$ ou $k=1)$, o resultado é

$$
F_{t}=0
$$

$$
F_{c}=m g\left(3 \cos \theta-2 \cos \theta_{o}\right) .
$$

Considerando um ponto que está próximo ao centro de oscilação, a quantidade $k$ é aproximadamente igual à 
unidade, ou seja, $k=1 \pm \varepsilon$. O símbolo $\varepsilon$ está associado com um pequeno deslocamento situado um pouco acima $(-\varepsilon)$ ou abaixo $(+\varepsilon)$ do centro de oscilaçã ${ }^{1}$ e as equações (19) e 21) resultam em

$$
F_{t}=\mp \varepsilon m g \operatorname{sen} \theta
$$

e

$$
F_{c}=m g\left((3 \pm 2 \varepsilon) \cos \theta-2(1 \pm \varepsilon) \cos \theta_{o}\right) \text {. }
$$

\subsection{Previsão sobre medidas com um acelerômetro solidário ao pêndulo}

Discutem-se a seguir as medidas que podem ser realizadas com um acelerômetro solidário ao pêndulo de tal forma que um de seus eixos coincide com o eixo radial e o outro com o eixo tangencial ao movimento do pêndulo.

Um acelerômetro sempre mede a intensidade de uma força por unidade de massa em cada um de seus eixos (força por unidade de massa no SI tem dimensão de newton por quilograma $(\mathrm{N} / \mathrm{kg})$ ou também metro por segundo ao quadrado $\left.\left(\mathrm{m} / \mathrm{s}^{2}\right)\right)$. É importante enfatizar que um acelerômetro não mede diretamente acelerações eventualmente ocorridas com ele, mas tais acelerações podem ser inferidas das medidas que ele é capaz de realizar. É uma obviedade que um acelerômetro meça força e nunca aceleração, pois todas as medidas são executadas no próprio sistema de referência do acelerômetro que, portanto, está em repouso em relação a si mesmo. Ou seja, no sistema de referência do próprio acelerômetro somente são possíveis medidas de deformação em pequenas molas (dinamômetros), levando à obtenção do valor de forças.

Se um acelerômetro se encontra estático no sistema de referência da Terra (considerado aqui como sendo o referencial inercial), ele mede as componentes da força da gravidade por unidade de massa em cada um dos seus eixos. A resultante das forças (por unidade de massa) medida é então aproximadamente $g \mathrm{~N} / \mathrm{kg}\left(\right.$ ou $\left.g \mathrm{~m} / \mathrm{s}^{2}\right)$. No entanto, se o acelerômetro estiver acelerado em relação à Terra, mede em cada eixo a composição da força da gravidade (por unidade de massa) com a força inercial (por unidade de massa), resultando em um valor diverso daquele que acontece quando o acelerômetro está em repouso (ou com velocidade constante) em relação à Terra.

Todavia, se um acelerômetro estiver localizado no centro de oscilação de um pêndulo, que oscila livre de resistência ou de qualquer outra força, exceto a da gravidade, deverá registrar um valor variável sobre o eixo orientado na direção radial e um valor nulo no eixo alinhado

\footnotetext{
${ }^{1} \mathrm{~A}$ quantidade $\varepsilon$ está associada com a dificuldade de experimentalmente localizar com precisão o centro de oscilação, podendo ser estimada empiricamente. Na verdade, como sabemos que a aceleração tangencial no centro de oscilação é nula, uma ótima estimativa para o valor de $\varepsilon$ é obtida pelo experimento, ou seja, este indicará quão próximo do centro de oscilação está o acelerômetro.
}

tangencialmente ao movimento do pêndulo, conforme demonstrado na seção anterior.

Caso o eixo radial do pêndulo tenha sido afastado da vertical por $\theta_{0}$, enquanto estiver em repouso, o acelerômetro fixado no pêndulo deverá registrar aproximadamente $g \cos \theta_{0}$ no eixo radial e $g \operatorname{sen} \theta_{0}$ no eixo tangencial, ou seja, somente as componentes da aceleração da gravidade. Entretanto, quando o pêndulo é liberado, o acelerômetro, fixado no centro de oscilação, deverá ter uma aceleração tangencial nula e uma aceleração radial dada pela equação 23 .

Como nosso objetivo é a análise das medidas obtidas por um acelerômetro, usando como aparato experimental algo similar a um balanço (Figura 1) ou, mais precisamente, o pêndulo mostrado na Figura 16, fixamos o acelerômetro próximo do centro de oscilação. Cabe salientar que, mesmo considerando a massa do assento/pêndulo muito maior do que a massa das cordas/pêndulo, a estimativa precisa do centro de oscilação não é uma tarefa simples, pois ainda envolve a distribuição de massa do assento/pêndulo. Outra dificuldade é a de alinhar os eixos do sistema de referência do acelerômetro com os eixos radial e tangencial do pêndulo. Todas essas dificuldades nos levam a estimar que o acelerômetro está em uma posição distante por $\pm \varepsilon$ do centro de oscilação.

Para exemplificar, analisaremos o caso particular de $\varepsilon=0,05$, isto é, o acelerômetro se situando um pouco acima do centro de oscilação $(k=0,95)$, ele registrará uma força tangencial por unidade de massa, em acordo com a equação (24,

$$
\frac{F_{t}}{m}=0,05 g \operatorname{sen} \theta
$$

inferior a $5 \%$ do valor da aceleração da gravidade mesmo que o pêndulo inicie sua oscilação com grande amplitude. Entretanto, no eixo radial, em acordo com a equação (25), o acelerômetro registrará uma força de vínculo radial ou centrípeta por unidade de massa dada por

$$
\frac{F_{c}}{m}=g\left(2,9 \cos \theta-1,9 \cos \theta_{o}\right)
$$

ou seja, praticamente o mesmo valor que registraria quando localizado no centro de oscilação.

Para construirmos um gráfico das medidas da força por unidade de massa nos dois eixos do acelerômetro em função de $\theta$ consideramos que o acelerômetro seja posicionado próximo ao centro de oscilação $(0,05 \mathrm{~m}) \mathrm{e}$ o eixo radial do pêndulo tenha sido afastado da vertical por um ângulo de $35^{\circ}$. Enquanto o acelerômetro estiver em repouso, em relação ao referencial inercial, deverá registrar aproximadamente $9,8 \cos 35^{\circ}=8,0 \mathrm{~N} / \mathrm{kg}$ no eixo radial e $9,8 \operatorname{sen} 35^{\circ}=5,6 \mathrm{~N} / \mathrm{kg}$ no eixo tangencial. O pêndulo, ao ser liberado, exibe uma aceleração em relação ao referencial inercial, entretanto, o acelerômetro que está solidário ao pêndulo, portanto localizado no referencial acelerado, medirá no eixo radial uma força 
por unidade de massa dada por

$$
\frac{F_{c}}{m}=28,4 \cos \theta-15,3
$$

enquanto, tangencialmente o acelerômetro deverá medir, em acordo com a equação (26), o valor de

$$
\frac{F_{t}}{m}=0,49 \operatorname{sen} \theta \text {. }
$$

Cabe destacar que o modelo de pêndulo com pequena amplitude apresenta erro máximo de cerca de $2 \%$, no período de oscilação, para lançamentos iniciais que não ultrapassam a marca de $35^{\circ}[5]$.

A Figura 6 mostra o gráfico das funções que descrevem as previsões sobre os valores da força por unidade de massa no eixo radial e no eixo tangencial do acelerômetro. Dessa forma, o gráfico indica que o valor mínimo da força por unidade de massa no eixo radial do acelerômetro é aproximadamente $8,1 \mathrm{~N} / \mathrm{kg}$ ( ou $8,1 \mathrm{~m} / \mathrm{s}^{2}$ ) e o valor máximo $13,2 \mathrm{~N} / \mathrm{kg}$ (ou $13,2 \mathrm{~m} / \mathrm{s}^{2}$ ), enquanto no eixo tangencial ao movimento a força por unidade de massa será pequena comparada com os dados da direção radial.

Modelamos a evolução temporal da elongação angular desse pêndulo a partir de $\mathrm{t}=1,0 \mathrm{~s}$ considerando a aproximação harmônica, ou seja,

$$
\theta=0,61 \cos \left(\frac{2 \pi}{3,04}(t-1)\right)
$$

em que o período para uma amplitude angular inicial de $35^{\circ}$ (0,61 rad) é aproximadamente $3,04 \mathrm{~s}$, pois o pêndulo utilizado tem cerca de $2,3 \mathrm{~m}$ de comprimento (esse valor é tomado como sendo aproximadamente o comprimento do pêndulo simples equivalente ao pêndulo físico).

As equações 28 e 29 são então reescritas, respectivamente, como

$$
\frac{F_{c}}{m}=28,4 \cos \left\{0,61 \cos \left[\frac{2 \pi}{3,04}(t-1)\right]\right\}-15,2
$$

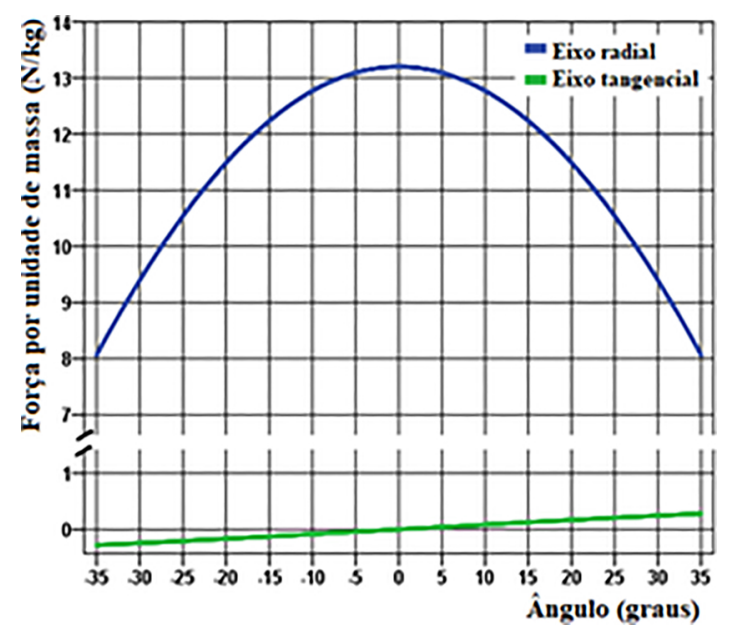

Figura 6: Previsão sobre as medidas da força por unidade de massa nos dois eixos do acelerômetro posicionado a $0,05 \mathrm{~m}$ do centro de oscilação.

$$
\frac{F_{t}}{m}=0,49 \operatorname{sen}\left\{0,61 \cos \left[\frac{2 \pi}{3,04}(t-1)\right]\right\} \text {. }
$$

O gráfico da Figura 7 representa os valores previstos teoricamente para as medidas realizadas com um acelerômetro preso a um pêndulo e na ausência de forças resistivas.

O gráfico da Figura 8 apresenta os valores registrados por um acelerômetro, posicionado aproximadamente 5 $\mathrm{cm}$ acima do centro de massa de um pêndulo com comprimento de $L \approx 2,3 \mathrm{~m}$ e na presença de forças dissipativas. O gráfico representa as medidas do acelerômetro no pêndulo em repouso durante o primeiro segundo já afastado $35^{\circ} \mathrm{em}$ relação à vertical. Posteriormente, em $\mathrm{t}=1,0 \mathrm{~s}$, o pêndulo é liberado e passa a oscilar.

O gráfico da Figura 7 prevê com boa aproximação as medidas efetivadas. Contudo, o modelo preditivo utilizado não inclui amortecimentos devido à dissipação de energia mecânica. Ao se comparar os dois gráficos (Figura 9) fica evidente que a amplitude das oscilações (Figura 8) diminui ao passar do tempo enquanto que o modelo teórico proposto não dá conta de tal aspecto.

Dessa forma, desconsiderando as perdas de energia, os resultados experimentais obtidos com o pêndulo estão em bom acordo com as previsões teóricas do modelo desenvolvido neste trabalho, como pode ser observado na Figura 9. Na próxima seção serão descritos em detalhes os processos que geraram os dados experimentais apresentados na Figura 8. Serão também apresentados resultados de medidas para outros valores de amplitude de lançamento.

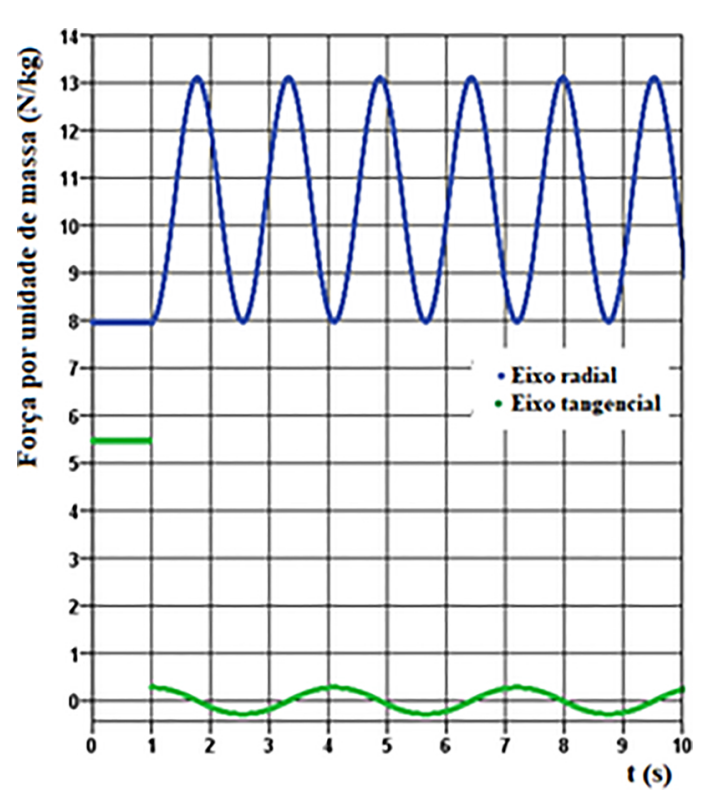

Figura 7: Valores previstos para as medidas com um acelerômetro nos dois eixos de um pêndulo (amplitude angular inicial de $35^{\circ}$ ( $\left.0,61 \mathrm{rad}\right)$ e com o acelerômetro posicionado aproximadamente a $0,05 \mathrm{~m}$ do centro de massa). 


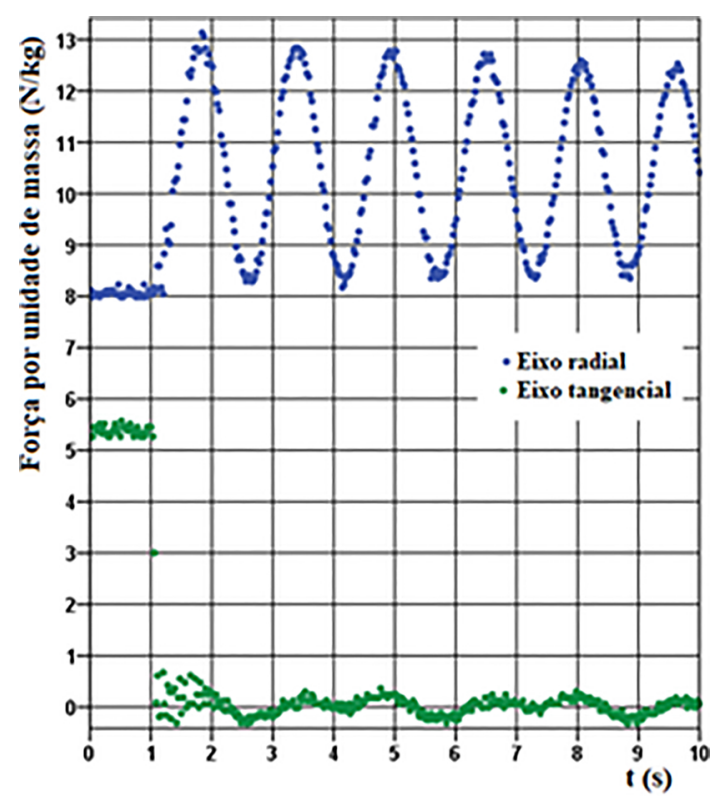

Figura 8: Valores medidos com um acelerômetro nos dois eixos de um pêndulo (amplitude angular inicial de $35^{\circ}$ (0,61 rad) e com o acelerômetro posicionado aproximadamente $0,05 \mathrm{~m}$ do centro de massa).

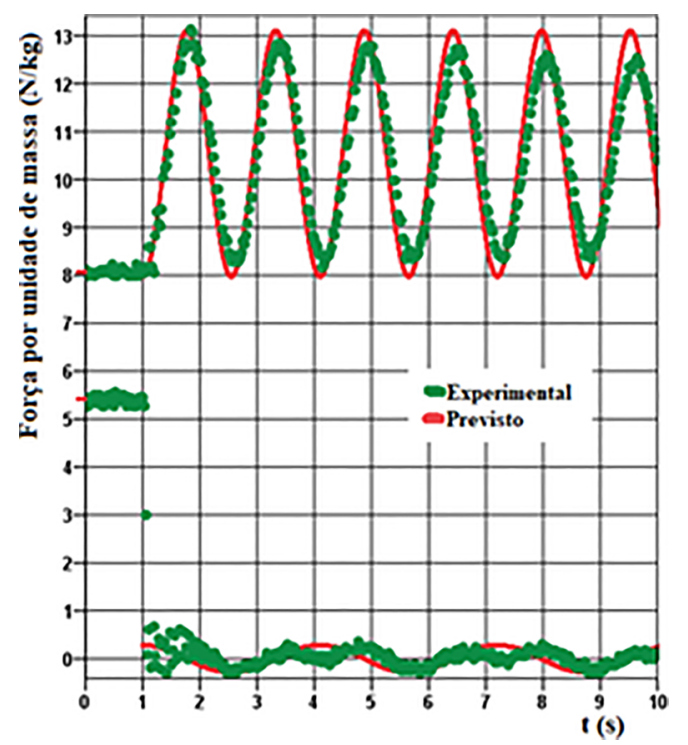

Figura 9: Sobreposição da curva teórica (vermelho) com os pontos experimentais (verde).

\section{Arduino, princípio de funcionamento do acelerômetro e descrição do aparato experimental}

Arduino é uma plataforma microcontrolada que possibilita o desenvolvimento de inúmeras tarefas, desde a realização de comandos simples, como piscar um LED, até tarefas mais complexas, como a automação de uma casa, por exemplo. Nesta proposta, utilizamos o Arduino como uma ferramenta programável de coleta de dados. O que define a função dessa placa é a forma como ela é pro- gramada, sendo a sua linguagem de programação baseada nas linguagens $\mathrm{C} / \mathrm{C}++$. Além disso, sua utilização vem ganhando espaço no desenvolvimento de novas tecnologias/funcionalidades, já existindo vários códigos prontos na Internet, permitindo que qualquer pessoa interessada possa utilizar o Arduino sem se preocupar em saber uma linguagem de programação. No presente trabalho, não nos deteremos em descrever as funcionalidades de cada componente da placa, como ligações e comandos. Porém, há um vasto material disponível na Interne ${ }^{2}$ que pode ser consultado.

Associado à placa Arduino, utilizamos o acelerômetro MMA7361 13 (Figura 10), responsável por fornecer um sinal de saída (uma diferença de potencial) que varia de acordo com a força sofrida pelo corpo de prova (com massa $m$ ), identificada esquematicamente na Figura 11. Tal figura foi adaptada do datasheet do fabricante, de modo a ficar ilustrada a presença de um corpo preso entre duas molas, o qual tem sua posição alterada pela componente da força da gravidade na direção paralela às molas e/ou por outras forças, inclusive forças inerciais, aplicadas diretamente no acelerômetro ou no aparato experimental em que se encontra o acelerômetro. Assim, é possível, conforme descrito na seção anterior, obter a força por unidade de massa sofrida pelo corpo de prova, uma vez identificada sua posição. Como não é possível observar diretamente a posição do corpo de prova, o funcionamento do acelerômetro utilizado (MMA7361) é

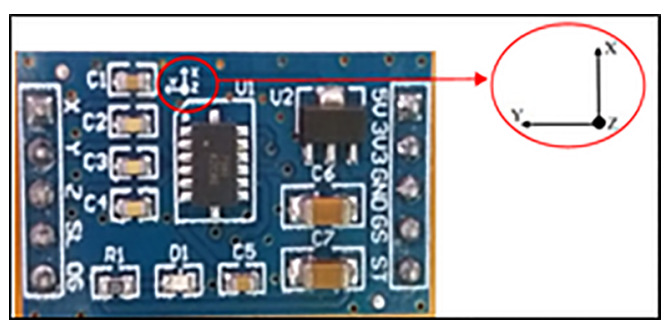

Figura 10: Acelerômetro (MMA7361), no detalhe eixos de orientação.

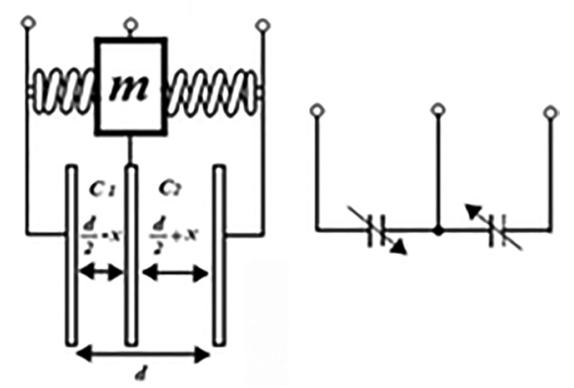

Figura 11: Representação esquemática de um acelerômetro. Fonte: Adaptação de https://www.nxp.com/docs/en/data-sheet/MMA7361L.pdf.

\footnotetext{
${ }^{2}$ https://www.arduino.cc/. Acessado em 15/08/2019.

${ }^{3}$ Datasheet disponível em https://www.nxp.com/docs/en/datasheet/MMA7361L.pdf Acessado em 15/08/2019.
} 
baseado na variação da capacitância que ocorre devido ao movimento de uma placa móvel (conectada rigidamente ao corpo de prova) entre duas placas fixas de um capacitor equivalente (Figura 11). Na verdade, o capacitor equivalente pode ser visto como uma associação em série de dois capacitores que possuem capacitâncias variáveis devido ao movimento de uma placa móvel. A placa central (móvel) tem sua posição alterada em função da presença de forças no corpo de prova. Dessa forma, cada conjunto de três capacitores (cada um associado a um eixo do acelerômetro), gera uma variação de capacitância, que produz uma diferença de potencial (sinal de saída), a qual pode ser relacionada linearmente com a força por unidade massa, ao ser fixado num objeto de interesse.

Para transformar o sinal de unidade de diferença de potencial do conversor analógico digital do Arduino em unidade de newton por quilograma $(\mathrm{N} / \mathrm{kg})$, posicionaramse os eixos $z$ e $y$ do acelerômetro, alinhados com a direção vertical, um de cada vez, e na sequência se orientaram tais eixos perpendicularmente à direção vertical, com o objetivo de determinar a diferença de potencial quando a componente gravitacional é nula nos eixos $z$ e $y$. Tais dados, depois de calibrados, geraram as seguintes equaçõe: 4

$$
\begin{aligned}
& \frac{F z}{m}=0,0605 *(\text { mediasensor } z)-16,212, \\
& \frac{F y}{m}=0.0613 *(\text { mediasensory })-21,989,
\end{aligned}
$$

em que mediasensorz(y) são as variáveis definidas no código de programação para fornecer as médias das leituras de diferença de potencial no eixo $\mathrm{z}(\mathrm{y}){ }^{5}$.

As equações (33) e (34) foram inseridas no código utilizado para programação da placa Arduino. Com base na Figura 11, fica ilustrado que a medida do acelerômetro está relacionada com a posição do corpo de prova e não com a variação da velocidade do acelerômetro (grandeza da cinemática) e a posição do corpo de prova, por sua vez, está relacionada com a deformação das molas. Assim, o presente trabalho refere-se às medidas do acelerômetro como uma medida de força por unidade massa, levando em conta as forças da gravidade e inerciais quando o acelerômetro se encontra em um referencial não inercial. As medidas realizadas com o acelerômetro permitem conhecer a sua aceleração em relação ao sistema de referência externo a ele (por exemplo, o sistema de referência do laboratório), considerado como um referencial inercial.

\footnotetext{
${ }^{4}$ Cabe salientar que se deve procurar alinhar ao máximo os eixos do acelerômetro com as direções desejadas para uma obtenção de dados mais próximos dos valores esperados. Outro fator importante é a fonte de alimentação, pois pode influenciar os valores de saídas do acelerômetro. Assim, sugere-se que a bateria de 9,0 V esteja conectada à placa Arduino no momento da obtenção das medidas. ${ }^{5} \mathrm{O}$ código utilizado está disponível em https://drive.google com/file/d/1Mdp0yLzKd43nqqAhGdYp-3K1vWgPYduw/view?usp= sharing Acessado em 15/08/2019.
}

Vieira e Aguiar [6] destacam que a aceleração medida por um acelerômetro é composta pela aceleração da gravidade, com direção e sentido dados pela orientação do acelerômetro, e pelos efeitos inerciais. Com o intuito de obter diretamente o valor da aceleração no sistema de referência inercial externo ao acelerômetro, Vieira e Aguiar [6] apontam a possibilidade de calibração do acelerômetro subtraindo da medida feita pelo acelerômetro o valor da aceleração da gravidade local, porém, alertando que, do ponto de vista analítico, isso só é possível em casos que não ocorrem rotações, pois, havendo rotações, as medidas nos eixos do acelerômetro estarão afetadas por forças inerciais decorrentes das próprias rotações.

O outro dispositivo eletrônico que foi utilizado é o módulo Bluetooth (Figura 12), que permite o transporte da informação, via radiação eletromagnética, entre a placa Arduino e o computador sem fazer uso de cabos elétricos, os quais poderiam causar alguma restrição no movimento a ser estudado. Essa comunicação sem cabos, incluindo o Bluetooth, evita tais inconvenientes interferências nos resultados. O módulo Bluetooth, foi montado em um circuito impresso, como mostra a Figura 13.

Esse aparato experimental, incluindo Bluetooth, acelerômetro e Arduino, foi utilizado para obtenção experimental do período de oscilação de um pêndulo. Portanto, o foco da proposta foi a coleta de dados usando a automação a partir do circuito eletrônico representado na Figura 14.

O aparato experimenta $\sqrt{6}$ formado por uma placa Arduino UNO, um módulo Bluetooth (HC-06), um acelerômetro (MMA7361) e uma bateria recarregável de 9,0 $\mathrm{V}$ foi montado em uma pequena base de madeira, com

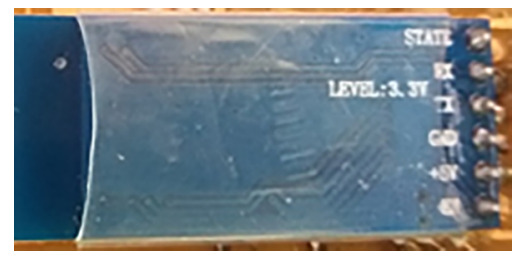

Figura 12: Módulo Bluetooth (HC-06).

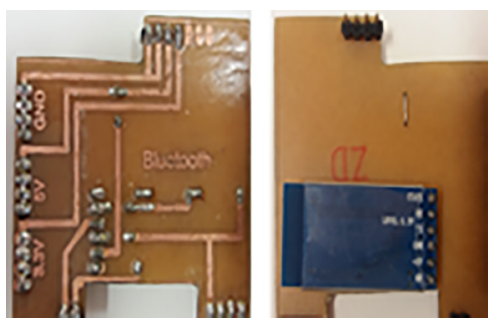

Figura 13: Circuito impresso do sensor Bluetooth.

\footnotetext{
${ }^{6} \mathrm{Um}$ passo a passo para a coleta de dados utilizando tal aparato (incluindo as etapas que devem ser desenvolvidas desde o pareamento do Bluetooth com o computador, a coleta de dados até a construção dos gráficos) está disponível em https://drive.google.com/file/d/1LftTGPBSwhsYAFzCW7nx8C_5BQeTN__/view?usp=sharing. Acessado em 15/08/2019.
} 


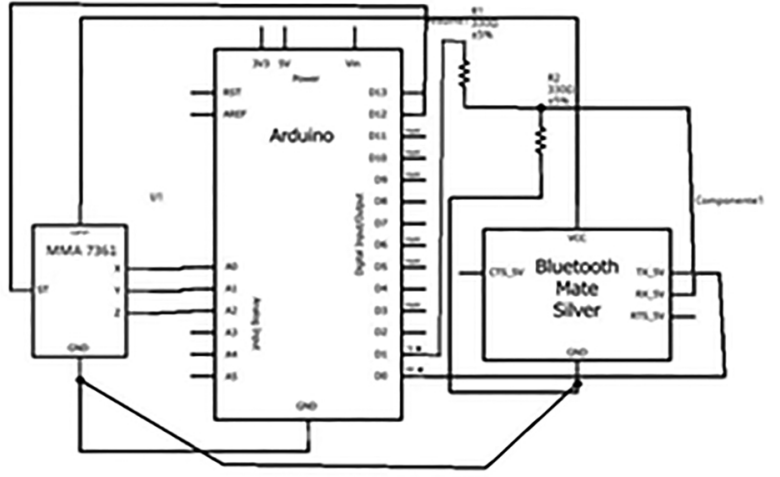

Figura 14: Circuito eletrônico do aparato experimental.

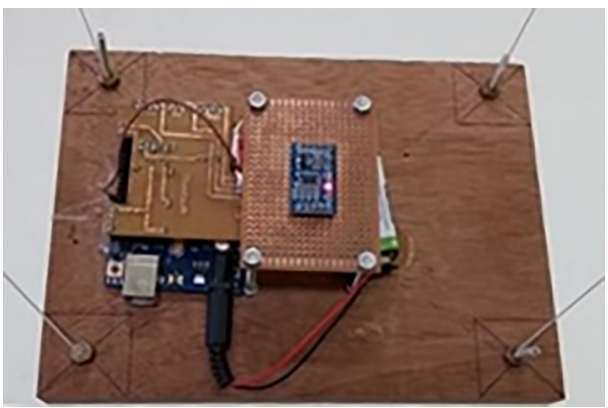

Figura 15: Imagem do aparato experimental.

dimensões de $15 \mathrm{~cm}$ por $20 \mathrm{~cm}$ (Figura 15). A massa total do conjunto é $519 \mathrm{~g}$, incluindo a massa de $3,4 \mathrm{~g}$ dos cordões, a massa da base de $410 \mathrm{~g}$ e 105,4 g dos equipamentos eletrônicos (Arduino, Bluetooth, acelerômetro, circuitos e bateria).

\section{O teste das previsões em um pêndulo com um acelerômetro}

O teste experimental para o modelo teórico até aqui apresentado foi realizado com um pêndulo de aproximadamente 2,3 $\mathrm{m}$ de comprimento. A base inferior do pêndulo é formada pela tábua em que foram fixados os componentes eletrônicos descritos na seção anterior. Nas arestas da tábua foram colocados parafusos ocos para facilitar a realização do nivelamento dela. Para a elevação da placa oscilante, utilizamos quatro cordões presos em dois pontos na parte superior (eixo de rotação) e, na inferior, aos quatro parafusos (Figura 16). Dessa forma foi possível minimizar movimentos na direção perpendicular ao plano formado pelos eixos $z$ e $y$ do acelerômetro, possibilitando a aproximação do movimento em duas dimensões apenas. Para identificação do ângulo de lançamento foi utilizado o transferidor posicionado próximo ao eixo de rotação, mas tal aferição foi comparada com uma medida de ângulo inserida no código de programação. Uma vez identificado o ângulo, deve-se ter muita atenção para soltar o pêndulo, pois os quatro cordões de

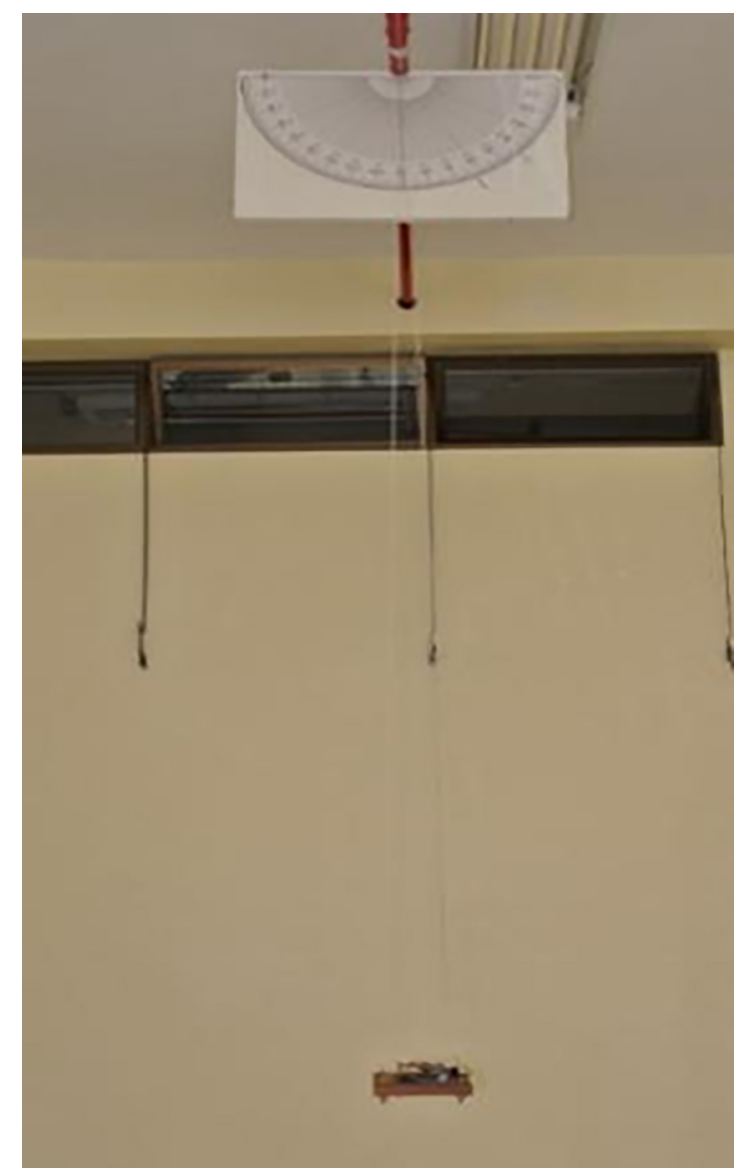

Figura 16: Imagem do aparato experimental posicionado paralelamente ao eixo $\mathrm{OQ}$.

sustentação devem estar com a mesma tensão e o balanço não pode iniciar a oscilação com vibração.

\subsection{O teste das previsões sobre as medidas de força por unidade de massa}

Com o objetivo de ilustrar as concordâncias entre as previsões teóricas do modelo desenvolvido na seção 2 e os resultados experimentais, construímos a Tabela 1. Na segunda e terceira coluna são apresentados os dados teóricos e experimentais para os valores máximos das medidas de força por unidade de massa na direção radial (instantes em que o eixo OQ fica alinhado com a direção vertical), enquanto nas duas últimas colunas é apresentado o erro relativo a cada medida (diferença entre o valor esperado e o medido) e o erro percentual. Conforme esperado, as diferenças entre os valores teóricos e experimentais aumentam à medida que se aumenta o ângulo de lançamento, pois o modelo teórico é construído com base em um sistema livre de resistência e os efeitos de forças resistivas são mais significativos nos casos com maior velocidade.

Os dados teóricos da Tabela 1 foram obtidos a partir da equação 27 , substituindo o valor nulo para $\theta$ (posição 
Tabela 1: Resultados teóricos e experimentais sobre os valores máximos das medidas de força por unidade de massa na direção radial.

\begin{tabular}{lcccc}
\hline Ângulos de lançamento & Teórico $(\mathrm{N} / \mathrm{kg})$ & Experimental $(\mathrm{N} / \mathrm{kg})$ & Erro & Erro percentual aproximado \\
\hline $15^{\circ}$ & 10,47 & 10,53 & $-0,06$ & $-1 \%$ \\
$25^{\circ}$ & 11,64 & 11,92 & $-0,28$ & $-2 \%$ \\
$35^{\circ}$ & 13,34 & 13,07 & 0,27 & $2 \%$ \\
$45^{\circ}$ & 15,54 & 15,01 & 0,53 & $3 \%$ \\
$55^{\circ}$ & 18,16 & 17,24 & 0,92 & $5 \%$ \\
$65^{\circ}$ & 21,12 & 18,82 & 2,30 & $11 \%$ \\
\hline
\end{tabular}

angular que coincide com o máximo da previsão das medidas na direção radial) e em $\theta_{o}$ o ângulo de lançamento.

\subsection{Obtenção experimental do período de oscilação}

Adicionalmente, as medidas obtidas com o pêndulo também permitem determinar o período das oscilações. Observe, na Figura 7 ou 8, que, enquanto o pêndulo realiza uma oscilação completa, a mola do acelerômetro alinhado com o eixo OQ mede duas oscilações completas, pois o intervalo de tempo entre os valores mínimos no gráfico da Figura 8 corresponde a uma oscilação completa da massa de prova do acelerômetro e a meio período de oscilação do pêndulo. Assim, podemos afirmar que se o período do pêndulo é $T$, então o período da massa de prova do acelerômetro é $\frac{T}{2}$, o que explica a ocorrência de três pontos máximos ou mínimos consecutivos dos valores medidos no eixo OQ ou radial durante uma oscilação do pêndulo.

Para obtenção do período de oscilação em função da amplitude inicial foram realizados três lançamentos com ângulos iniciais de $15^{\circ}, 25^{\circ}, 35^{\circ}, 45^{\circ}, 55^{\circ}$ e $65^{\circ}$. Os dados de tais lançamentos foram coletados pelo aparato experimental (Figura 15) e filmados para possibilitar a comparação entre duas técnicas. Em ambas as técnicas, em cada um dos três lançamentos foram medidos os intervalos de tempo de três oscilações iniciais, conforme Tabela 2 .

Nos gráficos gerados pelos dados com as medidas do acelerômetro $\left(\mathrm{Tec}_{1}\right)$, o intervalo de tempo inicial adotado constitui-se no ponto correspondente ao primeiro máximo da medida do eixo OQ (posição angular nula) e nos gráficos da videoanálise $\left(\mathrm{Tec}_{2}\right)$ utilizamos o ponto correspondente à realização da primeira meia oscilação. Assim, descartamos os instantes iniciais do movimento onde acontece muito ruído.

No gráfico da Figura 17 são apresentados os valores médios do período de oscilação (Tabela 2) do pêndulo de laboratório didático por meio do uso do acelerômetro (Tec1(s) - pontos na cor azul) e da técnica de videoanálise (Tec2(s) - pontos na cor verde). Confrontando esses resultados, encontra-se uma excelente concordância, ou seja, a diferença entre os períodos obtidos por meio das duas técnicas é menor que $1 \%$.

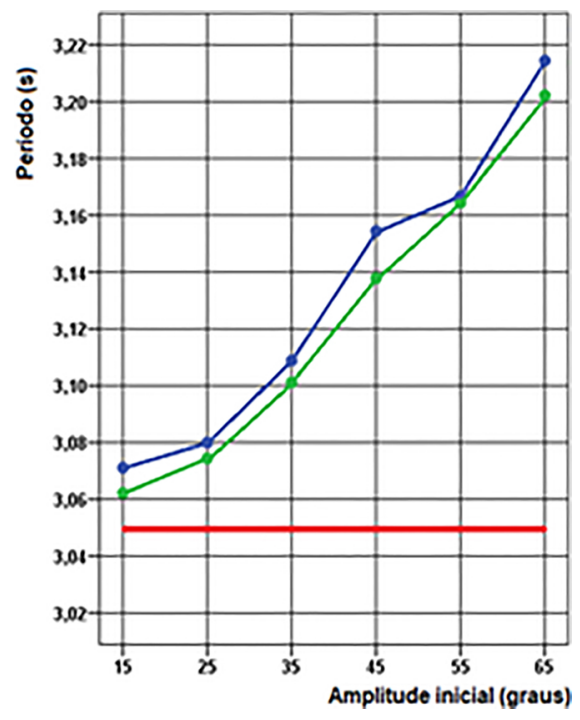

Figura 17: Análise gráfica dos dados experimentais sobre o período de oscilação do pêndulo.

Tabela 2: Dados experimentais sobre o período de oscilação do pêndulo.

\begin{tabular}{lcccccc}
\hline \multirow{2}{*}{ Medidas } & \multicolumn{2}{c}{$15^{\circ}$} & \multicolumn{2}{c}{$25^{\circ}$} & \multicolumn{2}{c}{$35^{\circ}$} \\
\cline { 2 - 7 } & $\mathrm{Tec}_{1}$ & $\mathrm{Tec}_{2}$ & $\mathrm{Tec}_{1}$ & $\mathrm{Tec}_{2}$ & $\mathrm{Tec}_{1}$ & $\mathrm{Tec}_{2}$ \\
\hline$\Delta \mathrm{t} 1(\mathrm{~s})$ & 9,23 & 9,22 & 9,23 & 9,22 & 9,34 & 9,34 \\
$\Delta \mathrm{t} 2(\mathrm{~s})$ & 9,21 & 9,15 & 9,24 & 9,24 & 9,31 & 9,28 \\
$\Delta \mathrm{t} 3(\mathrm{~s})$ & 9,20 & 9,19 & 9,25 & 9,21 & 9,33 & 9,29 \\
Valores médios & 3,07 & 3,06 & 3,08 & 3,07 & 3,11 & 3,10 \\
\hline Medidas & \multicolumn{2}{c}{$45^{\circ}$} & \multicolumn{2}{c}{$55^{\circ}$} & \multicolumn{2}{c}{$65^{\circ}$} \\
\cline { 2 - 7 } & $\mathrm{Tec}_{1}$ & $\mathrm{Tec}_{2}$ & $\mathrm{Tec}_{1}$ & $\mathrm{Tec}_{2}$ & $\mathrm{Tec}_{1}$ & $\mathrm{Tec}_{2}$ \\
\hline$\Delta \mathrm{t} 1(\mathrm{~s})$ & 9,45 & 9,37 & 9,50 & 9,45 & 9,63 & 9,59 \\
$\Delta \mathrm{t} 2(\mathrm{~s})$ & 9,46 & 9,47 & 9,52 & 9,50 & 9,63 & 9,61 \\
$\Delta \mathrm{t} 3(\mathrm{~s})$ & 9,48 & 9,40 & 9,48 & 9,53 & 9,67 & 9,62 \\
Valores médios & 3,15 & 3,14 & 3,17 & 3,16 & 3,21 & 3,20 \\
\hline & \multicolumn{5}{c}{}
\end{tabular}


Esses resultados, quando confrontados com o período teórico do pêndulo oscilando com pequena amplitude (T(s) - linha vermelha na Figura 17), corroboraram as previsões encontradas em [5] (apresentando desvios percentuais menores que $2 \%$ para ângulos de lançamentos menores que $35^{\circ}$ ). Desse modo, o pêndulo arquitetado pode ser descrito pelo modelo de pêndulo com pequena amplitude, com erro máximo de cerca de $2 \%$, para lançamentos iniciais que não ultrapassem a marca de $35^{\circ}$.

\section{Considerações finais}

Com base nos resultados obtidos neste artigo, agora somos capazes de responder às duas questões desafiadoras apresentadas no resumo. Para isso, no aparato mostrado na Figura 15 substituímos os componentes eletrônicos por a) um nível de pedreiro, onde a bolha de ar fica aproximadamente no centro de oscilação, e observamos que a bolha de ar fica estática enquanto o sistema oscila $7^{7}$ e b) uma esfera de aço (com diâmetro inferior a $3 \mathrm{~cm}$ ). Novamente, quando colocamos o sistema para oscilar, observamos que a esfera tende a ficar na posição central da tábua (centro de oscilação) na maioria dos lançamentos, exceto nos casos em que o balanço fica desalinhado ou a esfera e o balanço são soltos em tempos distintos.

Com base nos cálculos apresentados e nos dois experimentos descritos anteriormente, podemos concluir que, num sistema de referência não inercial de um pêndulo, qualquer objeto colocado no centro de oscilação está livre de uma força tangencial.

\section{Agradecimentos}

Ao prof. Edson Massayuki Kakuno e à profa. Eliane Angela Veit pelas inúmeras contribuições apresentadas durantes as etapas de coleta e análises dos dados experimentais.

Ao licenciando Ben-hur Martins Portella, que colaborou com o desenho da Figura 1.

Ao Projeto Feira de Ciências da Unipampa, Campus Bagé, pelo empréstimo dos componentes eletrônicos que foram adquiridos por meio de recursos do Conselho Nacional de Desenvolvimento Científico e Tecnológico - CNPq (chamadas CNPq/CAPES/MEC/MCTIC/SEPED).

\section{Referências}

[1] M.P.C. Moreira, M.C. Romeu, F.R.V. Alves e F.R.O. Silva, Caderno Brasileiro de Ensino de Física 35, 721 (2018).

[2] C.B.C. Silva, Uso da modelagem científica como recurso instrucional para o desenvolvimento de atividades expe-

\begin{tabular}{llllr}
\hline${ }^{7}$ Tais & experimentos & foram filmados e & estão \\
disponíveis & em & https://youtu.be/suvmPbu0rw8 & e \\
https://youtu.be/9gwZKIwcKrc Acessado em: & $15 / 08 / 2019$.
\end{tabular}

rimentais no Ensino Médio. Dissertação de Mestrado, Universidade Federal do Pampa, Bagé (2018).

[3] D. Halliday, R. Resnick e J. Walker, Fundamentos de Física: Mecânica (Rio de Janeiro, LTC, 2012), v. 1.

[4] K.R. Symon, Mechanics (Reading, Addison-Wesley, 1972).

[5] F.L. Silveira, CREF: Aproximação para a expressão do período de um pêndulo em pequenas amplitudes. Quais são os limites de validade da aproximação?, disponível em https: //www.if.ufrgs.br/novocref/?contact-pergunta= aproximacao-para-a-expressao-do-periodo-de-umpendulo-em-pequenas-amplitudes-quais-sao-oslimites-de-validade-da-aproximacao acessado em $13 / 02 / 2019$.

[6] L.P. Vieira e C.E. Aguiar, A Física na Escola 14, 8 (2016). 